indexed RV end-diastolic volume and body weight, whilst BNP was independently associated with VE/VCO2 $\left(\beta^{2}=0.8 ; 95 \% \mathrm{CI}\right.$ $0.1-1.6 ; \mathrm{p}<0.001)$, and to a lesser extent, anaerobic threshold $\left(\beta^{2}=-1.4 ; 95 \%\right.$ CI $\left.-2.2--0.6 ; p=0.001\right)$.

Conclusion In this cohort, BNP is independently associated with VE/VCO2, a marker of ventilation-perfusion mismatch, which is known to provide a more reliable assessment of exercise intolerance irrespective of effort level. These results therefore support a role for BNP as an useful marker of submaximal exercise capacity and for consideration in guiding prognosis.

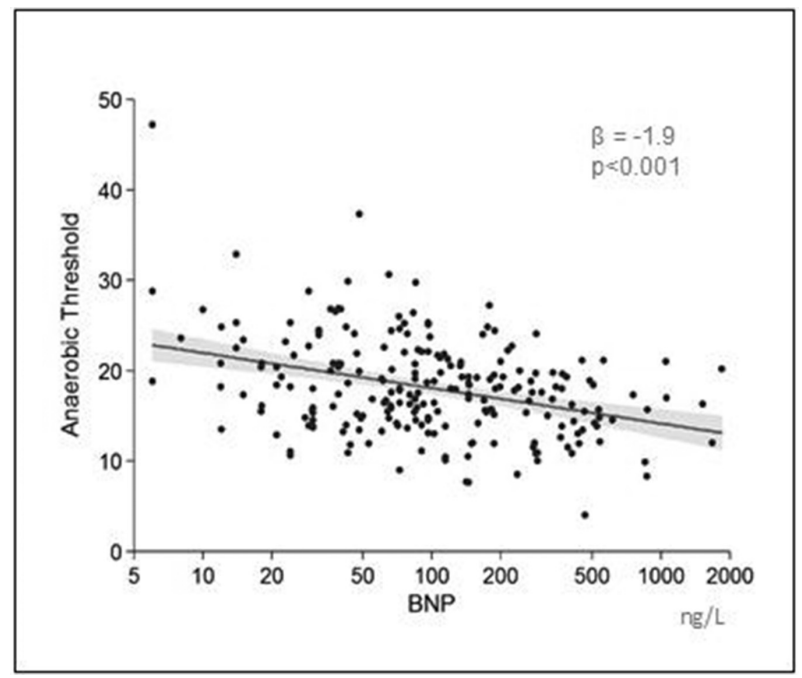

Abstract 127 Figure 2 Association between BNP and anaerobic threshold n HCM

\section{8 \\ GENOMIC INSIGHTS OF ECG STRAIN PATTEN IN AORTIC STENOSIS: T WAVE INVERSION AND ST-SEGMENT DEPRESSION ARE UNDERLINED BY DIFFERENT MOLECULAR PATHWAYS}

${ }^{1}$ Jiangting Hu*, ${ }^{2}$ John Pepper, ${ }^{1}$ Francesco Pezzella, ${ }^{3}$ Kevin Gatter, ${ }^{4} \mathrm{Xu}$ Yu Jin. ${ }^{1}$ Nuffield Division of Clinical Lab Sciences, Radcliffe Department of Medicine, University of Oxford; ${ }^{2}$ academic Cardiac Surgery, Royal Brompton Hospital; ${ }^{3}$ Nuffield Division of Clinical Lab Sciences, Radcliffe Department of Medician, University of Oxford; ${ }^{4}$ Department of Cardiothoracic Surgery, John Radcliffe Hospital

\subsection{6/heartinl-2017-311726.127}

Background ECG strain pattern is frequently presented in AS patients as a marker of LV hypertrophy and is emerging as one of the predictors for AVR. Our previous study had demonstrated that the normalisation of ST depression occurred within first 24 hours after AVR for AS while that of inverted $\mathrm{T}$ wave took $6-12$ months in parallel to the time course of $\mathrm{LVH}$ regression. We hereby studied myocardium gene expression profiling and tested the hypothesis that different molecular mechanisms could have underlined the ST depression and $\mathrm{T}$ wave inversion.

Material and methods We studied 17 AS patients with age 73 \pm 8.5 years and 12 males. LV biopsy was taken during AVR. Myocardial gene expression profiling was studied using Stanford Human Exonic Evidence Based Oligonucleotide (HEEBO) array. $\mathrm{T}$ wave inversion was defined by mean voltage of $\mathrm{T}$ wave in ECG lead I, aVL, V5 and V6 below $-50 \mathrm{uV}$ (T-invert, $\mathrm{n}=8$; T-norm, $\mathrm{n}=9$ ). ST depression was defined by a mean value of ST in the same leads being below $-50 \mathrm{uV}$ (ST-dep, $\mathrm{n}=9$; ST-norm, $\mathrm{n}=8$ ). Differential gene expression profiling within ST and T wave sub-group were carried out by parametric permutative (permutation times $=1000$ ) t-test using the $\mathrm{p}$ value cut-off 0.01 respectively. Further gene functional annotation clusters were performed using Database for Annotation, Visualisation and Integrated Discovery (DAVID, NIH) and pathway analysis of GeneSpring GX10.

Results We identified 588 differentially expressed genes (DEGs) between the T-invert and T-norm with 308 genes upregulated and 280 genes down-regulated in T-invert. Using Direct Interaction Network, genes with more intensive interactions were PLCL1, FGF1, MGP, FABP4, IL31 and IFI27 in up regulation and EGF, PAK2 SYT1, PPA1 and CSH1 in downregulation, in T-invert. Between ST-dep and ST-normal, we identified 202 DEGs, with 95 up and 107 down regulated in ST-dep. Using Direct Interaction Network analysis, we identified interactive connexion of genes, including ARHGEF1, ARRB1, PPM1A, CD28, SNX9, PRSS27, ASXL1, HDAC9 and BCOR, with no centre nodes orientation. The Significantly Enriched Core Pathways Analysis and DAVID gene functional annotation clustering further revealed that the top pathways underlined the genes of T-invert were oxidative phosphorylation, mitochondria, cell cycle/mitosis, cell adhesion, EGF/ERBB pathway, fatty acid metabolism; whilst for the genes of STdep, the main pathways were $G$ protein signalling pathway, transcription regulation of adipocyte differentiation and membrane trafficking/endocytosis.

Conclusions Our study provides new molecular insights into ECG strain pattern in LV hypertrophy due to AS. T wave inversion appears to be involved in down regulation of energy metabolism and cell cycle but ST-depression is mainly associated with changes in signalling transduction. Understanding these different biological significance will help a more precise clinical interpretation of ECG strain pattern and its changes following AVR for AS.

\section{ASSOCIATION OF ADVERSE VENTRICULAR REMODELLING AND GENDER IN AORTIC STENOSIS}

Anvesha Singh*, Daniel Chan, Leong Ng, Gerry McCann. University of Leicester

\subsection{6/heartjnl-2017-311726.128}

Introduction Previous echocardiography studies have demonstrated smaller left ventricles(LV) with more hypertrophy and concentric remodelling in females with Aortic Stenosis (AS) compared to males. However, more recent cardiac magnetic resonance(CMR) studies have shown lower LV mass and mass/ volume in females. We utilised CMR to assess myocardial perfusion and tissue characterisation, in addition to remodelling and function, and looked at biomarkers of fibrosis (Syndecan4 and MMP-3) in male and female patients.

Methods Subjects with asymptomatic moderate to severe AS ( 2 of: aortic valve area $<1.5 \mathrm{~cm}^{2}$, peak gradient $>36 \mathrm{~mm} \mathrm{Hg}$ or mean gradient $>25 \mathrm{~mm} \mathrm{Hg}$ ) were recruited in this prospective, multi-centre, observational study. All patients underwent venepuncture, echocardiogram and a comprehensive stress CMR.

Results 174 patients (133 male) were recruited. Females were slightly younger but there was no difference in resting haemodynamics, co-morbidities or AS severity between the genders (Table 1). Male patients had significantly higher LV volumes 
and mass, with more concentric remodelling, lower systolic (LV ejection fraction, longitudinal and circumferential global peak systolic strain) and diastolic function (longitudinal and circumferential peak early diastolic strain rates) than females. Late gadolinium enhancement (LGE) was more prevalent $(51.1 \%$ vs. $34.1 \%, \mathrm{p}=0.038)$ and extent of LGE was also higher in males, as was Syndecan-4 and MMP-3 levels. Extracellular volume (ECV) was marginally higher in female patients. Stress and rest myocardial blood flow (MBF) were significantly lower in males, with no difference in myocardial perfusion reserve.

Conclusions Male patients with AS have more concentric remodelling, worse cardiac function and more fibrosis than females, with biomarkers associated with fibrosis being significantly higher as well, for a similar degree of AS.

\begin{tabular}{llll}
\multicolumn{4}{l}{ Abstract 129 Table 1} \\
\hline & Male $(\mathrm{n}=133)$ & Female $(\mathrm{n}=41)$ & $\mathrm{p}=\mathrm{value}$ \\
\hline Age (years) & $67.3 \pm 12.64$ & $62.9 \pm 15.08$ & $0.042^{*}$ \\
Mean PG (mmHg) & $34.5 \pm 12.05$ & $38.0 \pm 13.66$ & 0.491 \\
AVAl $\left(\mathrm{cm}^{2} / \mathrm{m}^{2}\right)$ & $0.58 \pm 0.14$ & $0.55 \pm 0.15$ & 0.595 \\
LVEDVI $\left(\mathrm{ml} / \mathrm{m}^{2}\right)$ & $90.00 \pm 18.67$ & $79.74 \pm 14.50$ & $<0.001^{*}$ \\
LVEF $(\%)$ & $55.9 \pm 4.84$ & $59.2 \pm 4.49$ & $<0.001^{*}$ \\
LVMI $\left(\mathrm{g} / \mathrm{m}^{2}\right)$ & $60.54 \pm 13.70$ & $48.45 \pm 9.74$ & $<0.001^{*}$ \\
LV mass $/ \mathrm{volume}(\mathrm{g} / \mathrm{ml})$ & $0.68 \pm 0.11$ & $0.61 \pm 0.11$ & $<0.001^{*}$ \\
PSS $(\%)$ & $-17.85 \pm 2.80$ & $-20.52 \pm 2.81$ & $<0.001^{*}$ \\
PSS $_{C}(\%)$ & $-27.64 \pm 4.83$ & $-29.56 \pm 3.74$ & $0.002^{*}$ \\
\hline
\end{tabular}

\begin{tabular}{llll} 
Abstract 129 Table 1 & & \\
\hline & Male $(\mathbf{n}=133)$ & Female $(\mathbf{n}=41)$ & p-value \\
\hline Stress MBF $(\mathrm{m} / \mathrm{mm} / \mathrm{g})$ & $2.09 \pm 0.66$ & $2.39 \pm 0.80$ & $0.009^{*}$ \\
Rest MBF $(\mathrm{m} / \mathrm{min} / \mathrm{g})$ & $0.93 \pm 0.21$ & $1.14 \pm 0.36$ & $<0.001^{*}$ \\
Global MPR & $2.29 \pm 0.70$ & $2.18 \pm 0.70$ & 0.449 \\
$\%$ LGE (\%) & $4.6 \pm 3.87$ & $2.9 \pm 3.06$ & $0.001^{*}$ \\
ECV (\%) & $24.57 \pm 2.54$ & $25.64 \pm 1.85$ & $0.007^{*}$ \\
Log10.Syndecan-4 (pg/ml) & $2.33[$ IIQR 0.72] & $2.14[$ [IQR 2.39] & $0.044^{*}$ \\
Log10.MMP-3 (pg/ml) & $4.41[$ IIQR 0.88] & $4.24[$ [IQR 0.71] & $0.041^{*}$ \\
\hline
\end{tabular}

\section{THE CONVERGENCE AND DIVERGENCE OF MOLECULAR PATHWAYS IN LV HYPERTROPHY DEFINED BY ECG VOLTAGE VERSUS LV MASS IN PATIENTS WITH AORTIC STENOSIS}

${ }^{1}$ Jiangting Hu, ${ }^{2}$ John Pepper, ${ }^{3}$ Francesco Pezzella, ${ }^{4}$ Kevin Gatter, ${ }^{5} \mathrm{Xu}$ Yu Jin*. ${ }^{1}$ Nuffield Division of clinical lab Sciences, Radcliffe Department of Medician, University of Oxford; ${ }^{2}$ Academic Cardiac Surgery, Royal Brompton Hospital; ${ }^{3}$ Nuffield Division of Clinical Lab Sciences, Radcliffe Department of Medicine, University of Oxford; ${ }^{4}$ Nuffield Division of Cliinical Lab Sciences, Redcliffe Department of Medician, University of Oxford; ${ }^{5}$ Department of Cardiothoracic Surgery, John Radcliffe Hospital

\subsection{6/heartjnl-2017-311726.129}

Background Left ventricular hypertrophy (LVH) assessment by ECG voltage or image based mass volume is commonly used in clinical practice and research. Our previous study of LVH regression after AVR for AS demonstrated a more complete regression in ECG voltage than in LV mass, thus indicated that ECG and Echo could be quantifying different aspects of
LVH. In this study, we used gene expression profiling to examine whether different molecular pathways are involved in LV hypertrophy defined by ECG voltage or Echo LV mass.

Material and Methods We studied 17 patients with aortic stenosis, aged $73 \pm 8.5$ years with 12 males. Gene expression profiling of LV myocardium biopsy during AVR was carried out using Stanford Human Exonic Evidence Based Oligonucleotide (HEEBO) array. Using ECG QRS voltage cut-off $3.0 \mathrm{mv}, 8$ patients had LVH (ECG-LVH) and 9 had not (ECG-Norm ). Using Echo LV mass index $125 \mathrm{~g} / \mathrm{m} 2$ for man and $105 \mathrm{~g} / \mathrm{m} 2$ for woman, 9 cases had LVH (Echo-LVH) and 8 had not (Echo-Norm). The gene expression profiling comparisons were carried out between ECG-LVH vs. ECG-Norm as well as Echo-LVH vs. Echo-Norm, respectively, by parametric permutative (permutation times $=1000$ ) t-test using the $p$ value cut-off 0.01. Further gene functional annotation clusters were performed using Database for Annotation, Visualisation and Integrated Discovery (DAVID, NIH) and pathway analysed by GeneSpring GX10.

Results There are 163 genes significantly differentially expressed (DEGs) between ECG-LVH and ECG-Norm. Among which, 81 genes were up-regulated and 82 genes down-regulated in ECG-LVH. Between Echo-LVH and Echo-Norm, 256 DEGs were identified, of which 141 genes were up and 115 genes were down regulated in Echo-LVH. The Direct Interaction Network of 163 DEGs in ECG-LVH revealed several connected island hubs with genes of HGF, CDK5RAP2, FYN (up-regulated) and IL6, PTBP2 and IRF1 (down-regulated). Of 257 DEGs in Echo-LVH, there were more abundant islandlike connexions. The hubs of the connexion include PTEN, GNL3, AREG, ADAM17 and PPP1CA (up-regulated) and DCTN6, TWIST1 and ITGAM (down regulated). Further data mining and analysis of the two DEGs lists using DAVID and the Significantly Enriched Core Pathways analysis has demonstrated that the DEGs of ECG-LVH and ECHO-LVH have similar regulation directions in pathways/annotation clustering, including transcription regulation, nucleotide binding, zinc finger, focal adhesion. In addition, ECG-LVH has down regulation in wounding healing/inflammatory/cytokines pathway indicating a lower interstitial fibrosis activity compared with ECG-Norm; In Echo-LVH, mitochondrial protein importing, metalloprotease/ECM remodelling and cell division pathways were up-regulated compared to Echo-Norm.

Conclusions Cardiac hypertrophy diagnosed by ECG voltage or Echo muscle mass share many common molecular pathways that have been implicated in LVH. The unique pathways associated with ECG voltage based LVH imply a predominant hypertrophy of myocytes; in contrast, Echo mass based LVH involves the unique pathways of myocardial energy and ECM remodelling. The convergence and divergence of underlying molecular pathways between ECG based and Echo based LVH suggest the two methods may provide complimentary clinical value in the assessment of $\mathrm{LVH}$ and its response to treatment.

\section{LEFT VENTRICULAR INDEXED MASS ASSOCIATED WITH VENTRICULAR ARRHYTHMIAS IN PATIENTS WITH HYPERTROPHIC CARDIOMYOPATHY - A TERTIARY CENTRE MRI REGISTRY}

${ }^{1}$ Habib Khan*, ${ }^{1}$ Konstantinos Somarakis, ${ }^{2}$ Andrew Thain, ${ }^{1}$ Ayman Al-Atta, ${ }^{1}$ Thomas Mathew. ${ }^{1}$ Nottingham City Hospital; ${ }^{2}$ London Health Sciences Centre

10.1136/heartjnl-2017-311726.130 